\title{
LOS PROCESOS PARTICIPATIVOS COMO POTENCIADORES DE LA DEMOCRACIA. EXPLORANDO LOS EFECTOS, MECANISMOS Y EVIDENCIAS EN LA SOCIEDAD CIVIL'
}

\author{
Participatory processes as democracy makers. \\ Exploring effects, mechanisms and evidences \\ in civil society
}

\author{
PATRICIA GARCÍA-ESPÍN \\ Universidad Autónoma de Barcelona, \\ Consejo Superior de Investigaciones Científicas \\ pgarcia@iesa.csic.es \\ MANUEL JIMÉNEZ SÁNCHEZ \\ Universidad Pablo de Olavide \\ mijimsan@upo.es
}

Cómo citar/Citation

García-Espín, P. y Jiménez Sánchez, M. (2017).

Los procesos participativos como potenciadores de la democracia. Explorando los efectos, mecanismos y evidencias en la sociedad civil.

Revista de Estudios Políticos, 177, 113-146.

doi: https://doi.org/10.18042/cepc/rep.177.04

\section{Resumen}

¿Producen los procesos participativos los cambios democráticos que sugieren las aproximaciones normativas? $\mathrm{O}$, por el contrario, ¿tienen efectos negativos o resultan políticamente inocuos, como sugieren algunas investigaciones empíricas? Estas son cuestiones fundamentales que siguen, en gran medida, sin respuesta. El número de

Ambos autores han contribuido por igual en este artículo. Nos gustaría agradecer sus comentarios a Pau Alarcon, Laurence Bherer, José Luis Fernández, Ernesto Ganuza, Joan Font, Ank Michels y Graham Smith. También queríamos agradecer a los revisores la lectura cuidadosa del manuscrito. Este trabajo es parte del proyecto de investigación «Cherry-picking: The results of participatory processes», financiado por el Plan Nacional de Investigación del Ministerio de Economía y Competitividad (CSO2012-31832). 
efectos asociados a los procesos participativos parece ilimitado. Este trabajo recoge un marco de análisis para aclarar (parte de) este denso bosque de efectos. Para ello, realizamos una propuesta teórica para el análisis empírico. Además de la revisión de la literatura, el marco analítico se apoya en los discursos de expertos, académicos y profesionales de la participación pública. La propuesta se articula en torno a tres preguntas: ¿fomentan los procesos participativos la creación de capital social? ¿Favorecen el empoderamiento de los grupos asociativos como actores políticos? ¿Democratizan los patrones de interacción con las autoridades políticas? El trabajo aborda estas cuestiones desde un punto de vista teórico, proporcionando una clasificación de los efectos, mecanismos causales e indicadores de sus manifestaciones empíricas para pensar sobre las consecuencias de los procesos participativos en la sociedad civil y su interacción con el Estado.

\section{Palabras clave}

Democracia participativa; participación política; consecuencias de la participación; sociedad civil; capital social; empoderamiento; autonomía política; cooptación política.

\section{Abstract}

Do participatory processes produce democratic change as envisaged in normative accounts? Or, on the contrary, do they have negative effects or produce politically innocuous results, as some empirical research suggests? These are critical questions that remain largely unanswered. The potential effects associated with these processes seem boundless. This work seeks to clarify (part of) this vast forest of effects and articulate an analytical framework for empirical analysis. In addition to a literature review, the analytical framework proposed also rests on the discourse of academic scholars and public participation professionals. The resulting framework is developed around three research questions: Do participatory processes foster social capital? Do they empower social groups as political actors? Do they democratize patterns of interaction with political authorities? The paper addresses these questions from a theoretical point of view. It provides a classification of potential effects, mechanisms, and forms of evidence for thinking about the consequences of participatory processes in civil society and their interactions with the State.

\section{Keywords}

Participatory democracy; political participation; consequences of participation; civil society; social capital; empowerment; political autonomy; political cooptation. 


\section{SUMARIO}

I. INTRODUCCIÓN. II. LOS PROCESOS PARTICIPATIVOS COMO POTENCIADORES DE LA DEMOCRACIA: IDENTIFICANDO LOS PRINCIPALES EFECTOS EN LA LITERATURA. III. CONSULTANDO A LOS EXPERTOS PARA ACLARAR EL BOSQUE DE EFECTOS. IV. UN MARCO PARA ANALIZAR LOS EFECTOS DE LOS PROCESOS PARTICIPATIVOS EN LA SOCIEDAD CIVIL Y SU INTERACCIÓN CON LAS AUTORIDADES PÚBLICAS. H1. La hipótesis del arrecife de coral: los procesos participativos como factor de atracción de (nuevos) grupos sociales y aumento de interacciones. H2. La hipótesis del empoderamiento de la sociedad civil: procesos participativos como fortalecedores de la organización social. H3. La hipótesis del cambio en las relaciones de poder: el proceso participativo como transformador de los patrones de interacción dominantes. V. CONCLUSIONES. BIBLIOGRAFíA.

\section{INTRODUCCIÓN}

Los procesos participativos (PPs) son reformas institucionales que buscan ampliar las oportunidades de los ciudadanos para influir en las decisiones públicas. Entre las justificaciones para su implementación, a menudo encontramos argumentos sobre una posible profundización democrática (Gaventa, 2006; Fung, 2009). En este sentido, los PPs se consideran herramientas para fortalecer la sociedad civil, el empoderamiento de los grupos asociativos y el establecimiento de pautas de interacción con el Estado basadas en la autonomía.

En muchas de estas aproximaciones encontramos la idea de que las instituciones son, en gran medida, determinantes de la política (Cohen y Rogers, 1995; Lowi, 1972; Heinelt, 2007; Hall, 1986; Thelen, 1999). De manera optimista quizá, las reformas participativas se consideran como herramientas para transformar las relaciones de poder entre la sociedad civil y las autoridades, redistribuyendo el poder. No obstante, a medida que nos alejamos de los posicionamientos más normativos, algunos autores tienden a mostrarse escépticos acerca de la posibilidad de que tales efectos democratizadores tengan lugar. Consideran que, en no pocas ocasiones, los dispositivos participativos pueden responder (intencionalmente o no) a estrategias de movilización del sesgo para el mantenimiento del statu quo (Bachrach y Baratz, 1962; Navarro, 1999), reforzando patrones de interacción basados en la exclusión, la cooptación o la instrumentalización de actores sociales. 
En cualquier caso, el hecho es que, a pesar de la extensión de los procesos participativos en todo el mundo ${ }^{2}$, todavía sabemos poco sobre la realización efectiva de sus promesas de democratización. Las evidencias empíricas sobre sus efectos son limitadas, y no permiten extraer conclusiones definitivas ${ }^{3}$. A pesar de algunas excepciones (Nabatchi et al., 2012; Talpin, 2011; Funes et al., 2014; Blanco y Ballester, 2011; Baiocchi et al., 2011), carecemos de propuestas analíticas sistemáticas que delimiten y operacionalicen la larga lista de sus potenciales efectos en la sociedad civil y en la interacción con el Estado.

En este trabajo pretendemos desarrollar una propuesta de marco analítico para la identificación empírica de algunos de los supuestos efectos de los PPs relativos a la sociedad civil y su interacción con las autoridades. Esto significa que nuestro propósito, más que empírico, es principalmente de tipo teórico o conceptual: estamos interesados en la operacionalización de estos efectos como variable dependiente. Y aunque estamos interesados en los mecanismos causales detrás de estos efectos, no pretendemos explicar el grado de éxito o fracaso de los PPs.

Para elaborar nuestra propuesta de marco analítico seguimos una estrategia deductiva e inductiva en dos pasos. En primer lugar, se llevó a cabo una revisión de la literatura para delimitar el «subcampo» de los efectos de los PPs sobre la sociedad civil y en su interacción con las autoridades. Esta revisión teórica nos llevó a centrarnos en tres cuestiones interrelacionadas: ¿̇generan los procesos participativos capital social? ¿Empoderan a los grupos sociales como actores políticos? ¿Democratizan los patrones de interacción con las autoridades? Nuestro objetivo no está orientado a responder a estas preguntas sino a componer un marco analítico para su investigación empírica ${ }^{4}$. Para operacionalizar estos efectos, complementamos la revisión bibliográfica con

2 Véanse Ganuza y Baiocchi (2012); Smith (2009); Nabatchi et al. (2012), y Font et al. (2014).

3 Aunque los PPs han sido ampliamente estudiados para determinar, por ejemplo, la variedad de dispositivos (Fung, 2006), el tipo de prácticas que implementan (Font y Alarcón, 2014) y los aprendizajes que producen entre los participantes individuales (Talpin, 2011; Funes, Talpin y Rull, 2014), la atención a las transformaciones en la interacción entre la sociedad civil y las autoridades es más incipiente (Fung, 2009; Blanco y Ballester, 2011; Baiocchi et al., 2011).

4 Aunque el abanico de efectos posibles delimitado por las tres preguntas anteriores es muy amplio, aún deja de lado muchos otros efectos potenciales de los PPs. Cuestiones como la eficacia de las decisiones y la justicia social no se abordan en este trabajo. Como se observa en las secciones siguientes, también limitamos nuestra atención a los efectos que muestran empíricamente en un nivel meso de análisis, es decir, en el campo de la configuración organizativa de la sociedad y sus interacciones con el 
los resultados de una exploración de los discursos de expertos españoles. El objetivo es establecer un marco analítico útil para los investigadores así como para los profesionales interesados en elaborar protocolos para la evaluación de este tipo de impactos.

En la siguiente sección desarrollamos la definición de nuestro objeto de estudio: los efectos (democratizadores) de los PPs sobre la sociedad civil y su interacción con las autoridades. En la segunda sección detallamos la estrategia exploratoria, que fue determinante para recoger las opiniones de los expertos y afinar en la identificación de estos efectos y sus mecanismos causales. Por último, se presenta una propuesta de marco analítico que incluye 13 efectos diferentes agrupados en torno a tres hipótesis teóricas.

\section{LOS PROCESOS PARTICIPATIVOS COMO POTENCIADORES DE LA DEMOCRACIA: IDENTIFICANDO LOS PRINCIPALES EFECTOS EN LA LITERATURA}

Una cantidad considerable de argumentos que justifican la introducción de PPs proviene de la teoría de la democracia (Pateman, 1970; Cohen y Arato, 1992; Fishkin, 1997; Dryzek, 2000). El punto de partida es la reflexión sobre los profundos déficits de las democracias representativas: la introducción de mecanismos participativos se entiende como parte de un proceso más amplio de profundización democrática, concibiéndolos como complementarios y revitalizadores de los canales representativos existentes (Barber, 2003; Wampler, 2012; Warren, 2009). Además, los factores que en otros enfoques tienden a ser considerados como requisitos o condicionantes para el éxito de los PPs, aquí, se convierten en objetivos de la reforma institucional. Es decir, las condiciones del contexto político (por ejemplo, la densidad de la red de actores sociales o la naturaleza de sus relaciones con las autoridades políticas) deben modificarse mediante la implementación de los PPs. Así, las propiedades de una sociedad civil «fuerte», estructural (medida por su densidad organizacional y grado de conectividad) y culturalmente (medida por actitudes como la propensión hacia el bien público), puede ser no solo un prerrequisito para el éxito de los PPs, sino consecuencia de la gobernanza participativa (Kinney, 2012) .

Estado, excluyendo así de nuestro enfoque efectos observables en niveles macro (o agregados) y micro (o individuales).

5 Como señalan Fagotto y Fung (2009: 15), «aquellos que construyen instituciones y prácticas de participación pública frecuentemente trabajan en dos niveles. No sólo responden a las necesidades urgentes de sus comunidades, sino que, aunque no sea su intención, también mejoran la maquinaria del autogobierno democrático». 
Explícitamente o no, la mayoría de estos enfoques persiguen, en términos normativos, una reorientación en los patrones de interacción entre la sociedad civil y el Estado hacia relaciones de poder positivas, equilibradas y constructivas (Baiocchi et al., 2011; Montambeault, 2011). Por ejemplo, Evans (1996), a partir de diversos estudios de iniciativas de desarrollo económico en países en desarrollo, destacó como factor de éxito las sinergias creadas entre la sociedad civil y el Estado. En su estudio, los casos más destacables son aquellos en los que las relaciones se configuraron en redes que permean la división público-privado, sobre la base de relaciones de confianza y colaboración. Del mismo modo, Fagotto y Fung (2006) explican cómo el Programa de Revitalización de Minneápolis promovió la autonomía en las organizaciones (descentralizando la responsabilidad de la toma de decisiones a nivel de vecindario) y también promovió el empoderamiento, entendido como la transferencia de capacidades relevantes en forma de recursos, oportunidades de participación y capacidad decisional. Una conclusión similar puede encontrarse en el estudio de Abers (1998) sobre el presupuesto participativo en Porto Alegre.

Junto a la idea de sinergia, Evans (1996) asumió la idea de la «constructividad» del capital social ${ }^{6}$, encontrando suficiente evidencia para destacar el rol del Estado (las instituciones políticas) como promotor de la estructura organizativa de la sociedad civil, transformando o creando recursos organizativos (a través de la movilización de los lazos sociales existentes, la articulación de los recursos relacionales, etc.). Aplicada a la literatura de la gobernanza participativa, la reforma institucional podría desempeñar este papel de potenciador de la acción colectiva y fortalecimiento de la sociedad civil, tanto en su base organizativa como en su relación con las autoridades (Cohen y Rogers, 1995), transformando relaciones clientelares en relaciones basadas en la autonomía de los grupos asociativos.

6 En este sentido, véase también el trabajo de Wampler (2012), comparando estos efectos causados por la implementación de presupuestos participativos en diferentes distritos de Belo Horizonte. Incluso en las investigaciones empíricas en las que la presencia previa de actores organizados y autónomos se analiza como condiciones para el éxito, también se consideran los procesos participativos como potenciadores de las organizaciones y como oportunidades para formas de interacción políticamente relevantes basadas en la confianza y la colaboración. Véanse también el trabajo de Baiocchi et al. (2011) sobre el presupuesto participativo en Brasil y el de Abers (2007) sobre el establecimiento de consejos de cuencas hidrográficas también en Brasil. Estos hallazgos van en contra de la perspectiva de Putnam (Putnam et al., 1994), que considera el capital social en una sociedad dada como un factor exógeno, fuertemente arraigado a la cultura y, por consiguiente, difícil de cambiar a corto plazo (como ejemplos de enfoques críticos véanse Tarrow, 1996; Jackman y Miller, 1998). 
En resumen, se considera que estos dispositivos participativos contribuyen a una variada gama de cambios positivos. Una gran cantidad de efectos giran en torno a la idea de los PPs como promotores del capital social (impulsando la acción colectiva y la coordinación en la sociedad civil), como factores de empoderamiento de los grupos asociativos (generando nuevos recursos) y fomentando nuevos modos de interacción con las autoridades (basada en la autonomía, la transparencia y la horizonalidad) (Fung y Wright, 2001; Baiocchi et al., 2011; Abers, 2003). En muchos casos, los PPs también se consideran como facilitadores de la inclusión de sectores excluidos (Wampler, 2007; Hernández-Medina, 2010).

Conforme a estos argumentos, hemos identificado tres hipótesis sobre los efectos potenciales de los PPs. En primer lugar, la hipótesis del «arrecife de coral» implica que los PPs fomentan el capital social ${ }^{7}$. De la misma forma que los arrecifes artificiales atraen a diferentes especies, proporcionando un ambiente protector para su desarrollo, los PPs pueden incentivar la acción colectiva, atraer a grupos asociativos o nuevos participantes, y aumentar los lazos entre ellos. Por lo tanto, en la medida en que favorecen la creación de redes y la confianza, los PPs pueden ser considerados como fuentes de capital social. En segundo lugar, la «hipótesis de empoderamiento de la sociedad civil» considera que los PPs implican nuevos recursos organizativos para los grupos asociativos y fomentan nuevas percepciones de eficacia política. Por último, bajo la hipótesis del «cambio en las relaciones de poder», los PPs son concebidos como transformadores de los modos establecidos de interacción en favor de nuevos modos basados en la autonomía de los grupos asociativos.

Sin embargo, las expectativas normativas expresadas en estas hipótesis parecen lejos de cumplirse en la práctica. A pesar de la importancia de los casos exitosos, habitualmente los logros democráticos de la mayoría de los PPs están lejos de las mejores prácticas. Por el contrario, parece que la realidad más común es la de la resistencia y la continuidad de las relaciones de poder establecidas (Ganuza et al., 2014; Walker et al., 2015). En su análisis de los consejos consultivos en municipios españoles, Navarro (1999) identificó, como práctica dominante, la selección de los participantes según las afinidades electorales del partido político titular. De manera similar, Parkinson (2004) mostró cómo, en un proceso de reforma de la política de salud en el Reino Unido, la participación se abrió a los ciudadanos no organizados como estrategia para excluir a los sectores más críticos y desafiantes. En ocasiones, la dinámica de

7 La metáfora del arrecife de coral ha sido tomada del trabajo de Tarrow (2001: 5) sobre la formación de redes trasnacionales de actores sociales en el entorno de las instituciones internacionales. 
trabajo de la administración, e incluso sus horarios, pueden resultar conflictivos con la participación (Sintomer y Ganuza, 2011) y la definición de los problemas puede reducir la ambición de la participación (Eliasoph, 1998). Como señalan Baiocchi et al. (2011) en su estudio comparativo sobre presupuestos participativos en Brasil, las configuraciones relacionales entre los actores asociativos y las autoridades (de la autonomía a la cooptación) dependen del empoderamiento previo de la sociedad civil y del diseño institucional. En definitiva, los PPs pueden ser considerados como oportunidades políticas para el empoderamiento de los ciudadanos y el fortalecimiento democrático de la sociedad civil, pero también pueden responder a lógicas de movilización del sesgo institucional, la instrumentalización y la legitimación del statu quo.

La realidad es que, de forma merecida o no, las expectativas sobre los PPs han sido enormes: un rápido vistazo a la literatura especializada es suficiente para descubrir una gran lista de potenciales efectos, sobre todo positivos. Nuestro objetivo consiste en aclarar (parte de) este extenso bosque de efectos, positivos o negativos, intencionados o no intencionados, y articularlos en una propuesta teórica para el análisis empírico.

\section{CONSULTANDO A LOS EXPERTOS PARA ACLARAR EL BOSQUE DE EFECTOS}

Los efectos de los PPs en la sociedad civil han sido abordados en el contexto de las reformas participativas españolas. La experiencia de procesos participativos en los últimos treinta años se desarrolla de manera paralela a otros países del sur de Europa (Navarro, 1999; Font, Della Porta y Sintomer, 2014). Por ejemplo, durante los años 2000, España registró una expansión extraordinaria de experiencias de presupuestos participativos (Ganuza y Francés, 2012). El momento de nuestro trabajo de campo (primavera de 2014) resultó particularmente propicio para indagar sobre los efectos de esta reforma institucional. El contexto de crisis económica y de retroceso en el gasto público conllevó la cancelación de muchos PPs. También se dio un aumento de las protestas sociales y el florecimiento de nuevos actores colectivos. Este escenario político resultó extremadamente estimulante para la reflexión sobre la democracia participativa.

Como hemos señalado, nuestra propuesta se centra en los efectos democratizadores de los PPs en la sociedad civil y su interacción con el Estado. La tarea de diseñar un marco analítico implicó, en primer lugar, identificar y definir los efectos, positivos o negativos, que pueden estar razonablemente asociados a los PPs. Por «razonablemente» nos referimos a efectos que son susceptibles de observación empírica (por ejemplo, traducibles a indicadores) 
y que pueden ser vinculados a PPs a través de un mecanismo explicativo plausible. En segundo lugar, también conllevó una agrupación de esos efectos bajo tres amplias hipótesis que permitieran, finalmente, guiar un análisis empírico sistemático. Además de la revisión de la literatura, nuestro trabajo se fundamenta en los resultados obtenidos en una serie de entrevistas con expertos.

Estas entrevistas fueron concebidas como una herramienta para ayudarnos a traducir los supuestos efectos normativos de los PPs en manifestaciones empíricas. Además, necesitábamos una inmersión realista en el campo porque los efectos mencionados en la literatura resultan innumerables y, con frecuencia, demasiado abstractos. Este trabajo exploratorio con expertos permitió concretar nuestra propuesta. Por un lado, los expertos contaban con la experiencia concreta para contrastar las afirmaciones normativas con evaluaciones más realistas que adquirieron en su desarrollo profesional ${ }^{8}$.

Realizamos entrevistas personales semiestructuradas con ocho académicos, reconocidos como especialistas en el campo de la participación ciudadana en España ${ }^{9}$, en tres comunidades autónomas diferentes para cubrir parte de la variedad territorial existente. También organizamos dos grupos nominales con profesionales de la participación pública en Andalucía y Cataluña ${ }^{10}$. Para las entrevistas y los grupos nominales utilizamos un guión similar con cuatro dimensiones de efectos: a) cambios organizacionales internos en los actores de la

8 Este tipo de trabajo de campo exploratorio resulta particularmente útil como primera inmersión en fenómenos que apenas se han estudiado. Se ha llamado exploración innovadora (Stebbins, 2001: 2). A pesar de que se ha subestimado con frecuencia, y solo se utiliza para mejorar la calidad de los diseños de investigación (Bergman y Coxon, 2005), el trabajo de campo exploratorio puede ser una excelente fuente de datos para la definición de marcos analíticos, la operacionalización de conceptos y la elaboración de protocolos de evaluación (Sieber, 1973). Resulta especialmente necesario para salvar la distancia entre constructos abstractos y normativos, y su traducción en un conjunto de manifestaciones empíricas concretas.

9 Los académicos entrevistados fueron: Tomás Alberich (Universidad de Jaén), Julio Alguacil (Universidad Carlos III, Madrid), Quim Brugué (Universitat Autònoma de Barcelona), Ernesto Ganuza (IESA-CSIC), Jaume Magre (Universitat de Barcelona), Carmen Navarro (Universidad Autónoma de Madrid), Clemente Navarro (Universidad Pablo de Olavide, Sevilla) y Fernando Pindado (Universidad Autónoma de Barcelona).

10 El concepto de "profesionales de la participación pública» ha sido propuesto por Bherer, Gauthier y Simard (2016). A partir de aquí, utilizaremos los términos «académicos» para referirnos a los entrevistados académicos y «profesionales» a los profesionales de la participación pública (es decir, al amplio espectro de facilitadores, asesores externos y funcionarios públicos que intervienen en el desarrollo de los PPs). 
sociedad civil, b) cambios en sus interacciones con los ciudadanos, c) en la red de grupos sociales, y d) en sus relaciones con las autoridades (véase el anexo).

Realizamos análisis temático (Boyatzis, 1998) de las entrevistas y grupos nominales para identificar los efectos priorizados por los expertos y los mecanismo causales (a modo de hipótesis plausibles). Al inicio de las entrevistas, los expertos académicos tendieron a mostrarse dubitativos, a menudo argumentando que el tema de los resultados había recibido escasa atención. Como explicó uno de los especialistas pioneros en el estudio de los PPs en España:

En la literatura, el tipo de análisis que estáis considerando no se ha realizado, hay estudios de caso que describen casos maravillosos que suceden en algún lugar, pero no hay evaluación después de cinco años o, incluso, seis meses. No hay estudios comparados que nos permiten llegar a conclusiones en este terreno (entrevista académico).

Respecto a los grupos nominales con profesionales, ofrecieron una descripción más detallada de los efectos, sobre la base de una experiencia más directa. Aquellos que basaban sus juicios en su experiencia en mecanismos participativos no permanentes o menos intensos (por ejemplo, foros deliberativos) tendían a mostrarse más pesimistas, mientras que los que referencian los presupuestos participativos (la mayoría en el grupo nominal de Andalucía) eran más propensos a identificar efectos positivos. Como explicó uno de estos profesionales:

El fortalecimiento del tejido asociativo me parece determinante: el fortalecimiento de las organizaciones existentes, la aparición de nuevas, la generación de contradicciones entre las existentes [...] En definitiva, a veces puede llevar a romper el muro [para cambiar] (profesional, GN-Andalucía).

La gama de efectos destacados por académicos y profesionales no siempre se superpone; ni tampoco coinciden sus evaluaciones sobre la ocurrencia efectiva de estos efectos. En general, los expertos profesionales tienden a poner más énfasis en los positivos. Además del potencial sesgo profesional y su frecuente compromiso normativo con la idea de participación (un potencial «efecto Pigmalión»), se puede argumentar también que sus experiencias en el día a día de los PPs aumentan su sensibilidad a efectos incipientes.

Además, la evaluación de los efectos depende de los PPs que los expertos conocen mejor: los mecanismos participativos específicos y el contexto político con el que están familiarizados. Entre los académicos, la referencia más común es la asociativa (y de manera particular los consejos asesores o consultivos), lo que sugiere puntos de vista bastante negativos sobre los modelos 
clientelares y los procesos de institucionalización asociativa tras la Transición, en la década de los años ochenta. Cuando las referencias son innovaciones participativas recientes (como el presupuesto participativo), las visiones negativas se matizan. En este sentido, los PPs pertenecientes a la ola más reciente de innovaciones participativas constituyen la principal referencia empírica entre los profesionales; para ellos, cuanto más intensa y duradera sea la participación, más positivos serán los efectos ${ }^{11}$.

Nuestra consulta a los expertos significó, en primer lugar, una oportunidad para completar nuestra lista de los efectos democratizadores de los PPs (particularmente identificando los efectos negativos, a menudo soslayados en la literatura), así como para priorizar los más relevantes. En segundo lugar, también fue de gran ayuda para confirmar algunos de los mecanismos causales. Y finalmente, contribuyó a mejorar nuestra comprensión de la naturaleza empírica de los efectos y a pensarlos en términos de indicadores. En general, los datos del trabajo exploratorio nos ayudaron a pensar en términos de efectos, mecanismos y evidencias, configurando la propuesta analítica que planteamos a continuación.

\section{UN MARCO PARA ANALIZAR LOS EFECTOS DE LOS PROCESOS PARTICIPATIVOS EN LA SOCIEDAD CIVIL Y SU INTERACCIÓN CON LAS AUTORIDADES PÚBLICAS}

La propuesta analítica resultante agrupa una selección de 13 efectos, tanto de carácter estructural como cultura ${ }^{12}$. Estos efectos se agrupan en tres

11 Los mecanismos participativos referidos por los expertos para explicar sus visiones y evaluaciones son: a) Reglamentos Participativos (Planes Participativos, Regulaciones Locales), mencionados por 6 académicos; b) Espacios de democracia asociativa (consejos temáticos y territoriales, consejos infantiles, consejos escolares, talleres para organizaciones cívicas, talleres participativos, foros), mencionados por 15 expertos; c) Planes Estratégicos (Inversiones Especiales, Planes Integrales, Planes de Vecindad, Obras Públicas, Planes de Acción, Planes Educativos, etc.), mencionados por 8 expertos; d) Presupuesto Participativo, mencionado por 11 expertos; e) otras prácticas (encuestas o jurados) y dispositivos basados en Internet, mencionados por 6 expertos.

12 Consideramos, aquí, dos dimensiones diferentes en las que los efectos pueden ser observados empíricamente. En primer lugar, la dimensión «estructural» se refiere a los recursos de las organizaciones, las relaciones entre los actores y las formas de organización. En segundo lugar, la dimensión «cultural» se refiere al conjunto de efectos que se ocupan de la percepción, los valores y las representaciones que tienen los actores. 
grupos, consistentes con las tres hipótesis generales. Más concretamente, un primer grupo se relaciona con la idea de que los PPs favorecen la acción colectiva (o el capital social) atrayendo y conectando a los actores asociativos. Un segundo grupo de efectos responde a la idea de que los PPs capacitan a los actores asociativos. Y un tercer grupo de efectos desarrolla la idea de que modifican los patrones generales de interacción entre la sociedad civil y las autoridades locales.

Como puede observarse en las siguientes tablas, para cada uno de estos trece efectos se proporcionan ejemplos de indicadores empíricos, y se sugieren mecanismos explicativos plausibles. Aunque las tres hipótesis se formulan en términos positivos, en su discusión se plantean, también, sus posibles efectos negativos.

\section{H1: LA HIPÓTESIS DEL ARRECIFE DE CORAL: LOS PROCESOS PARTICIPATIVOS COMO FACTOR DE ATRACCIÓN DE (NUEVOS) GRUPOS SOCIALES Y AUMENTO DE INTERACCIONES}

Es posible que en momentos de efervescencia la gente se haya articulado más por la propia inercia del proyecto, pero no ha cuajado, no ha quedado nada. Las asociaciones se mueven igual, a través de las subvenciones discrecionales de las administraciones. Tampoco creo que hayan aparecido nuevas a rebufo de estos procesos, pero de esto tenemos pocos datos (entrevista académico).

Los procesos participativos contribuyen a que [los grupos] se visualicen mutuamente [y aumenta el trabajo en red] (profesional NG-Andalucía).

¿Fomentan los procesos participativos la creación de capital social? De acuerdo con la idea de la "constructividad» del capital social (Evans, 1996), cabe suponer que los PPs establecen espacios institucionales (arenas políticas) que propician las interacciones y las iniciativas de coordinación entre individuos y actores asociativos que de otro modo serían difíciles. Por lo tanto, podemos pensar que funcionan en la sociedad civil como los «arrecifes de coral» lo hacen en la vida marina.

Desde una perspectiva estructural, los PPs pueden suponer un cambio en la estructura de costes y beneficios asociada a la acción colectiva, facilitando la formación de nuevos grupos o atrayendo nuevos participantes (tabla 1, efecto 1). Una vez resueltos los problemas iniciales de coordinación, los PPs pueden incrementar la probabilidad de que la acción colectiva se mantenga en el tiempo y tome cuerpo en grupos más o menos formalizados. Las expectativas de acceso a recursos (de influencia, por ejemplo) pueden actuar como un factor impulsor para grupos que no estaban previamente organizados. Así como los arrecifes de coral fomentan la diversidad de especies, el aumento en 
el número de grupos puede conducir a una mayor diversidad o pluralidad de participantes. Por el contrario, si los PPs son percibidos como costosos, las expectativas reducidas, o los resultados son frustrantes, los nuevos participantes potenciales pueden abstenerse y otros pueden abandonar (potencialmente, reduciendo la diversidad dentro de las redes de políticas). En algunas ocasiones, la salida puede implicar una forma de expresar desacuerdo o rechazo del nuevo escenario político (Hirschman, 1982).

La conectividad social y el funcionamiento de redes entre grupos puede activarse (efecto 2). En este caso, los PPs pueden activar mecanismos de intermediación entre grupos (no conectados previamente), ya que implican convocatorias más o menos amplias a la participación, promoviendo la convergencia $y$, eventualmente, futuras colaboraciones y acciones conjuntas ${ }^{13}$. Por el contrario, un sesgo en la selección de los participantes, intencionado o no, podría desencadenar un mecanismo de cierre, limitando el flujo de información e incluso reduciendo el universo de actores conectados.

Como ilustran las citas al principio de este epígrafe, los académicos y los profesionales tienden a tener opiniones diferentes sobre la ocurrencia de estos efectos. Los profesionales son más sensibles a la aparición de este tipo de efectos de «atracción» (tabla 1, efecto 1). Por lo general, ubican estos efectos en las experiencias de presupuestos participativos, con ejemplos positivos de confluencia de participantes anteriormente no organizadas, así como la incorporación de sectores previamente excluidos (de mujeres, inmigrantes, jóvenes, etc.). En contraste, los académicos tienden a ser escépticos. En su opinión (fundamentalmente basándose en su conocimiento de los consejos consultivos), el principal cambio en la estructura de costes y beneficios de la acción colectiva no es la introducción de PPs, sino el flujo (discrecional) de subvenciones públicas, a menudo, vinculadas a la prestación de servicios.

Curiosamente, ambos tipos de expertos también se refieren a la ocurrencia de casos de desconexión y salida de grupos asociativos. En algunos casos, la falta de resultados tangibles (falta de beneficios y frustración) puede llevar a algunos actores a abandonar. Los expertos conectan estos efectos con percepciones de irrelevancia política y discontinuidad de los PPs ${ }^{14}$. Es decir,

13 Como señalan dos profesionales: «Los PPs contribuyen a que los grupos se visualicen mutuamente y y aumenta el trabajo en red» (GN profesionales-Andalucía). «El proceso participativo facilita un espacio que en otras circunstancias, esas personas jamás se hubieran relacionado [...] no solo a nivel individual sino [posteriormente] en movimientos, plataformas y en mareas» (GN profesionales-Cataluña).

14 Algunos profesionales también mencionan como factor de fatiga participativa y abandono la rutinización de las herramientas participativas, su falta de innovación y adaptación a las formas de interacción habituales de las personas. 
Tabla 1. La hipótesis del arrecife de coral: los procesos participativos atraen (nuevos) grupos sociales e incrementan sus conectividad

\begin{tabular}{|c|c|c|c|}
\hline Nivel & Efectos & $\begin{array}{l}\text { Manifestaciones empíricas } \\
\text { (ejemplos) }\end{array}$ & $\begin{array}{l}\text { Mecanismos explicativos } \\
\text { (sugeridos) }\end{array}$ \\
\hline \multirow{4}{*}{ 焉 } & \multirow[t]{2}{*}{$\begin{array}{l}\text { (1) } \\
\text { Atracción } v s . \\
\text { salida }\end{array}$} & $\begin{array}{l}\text { Aumento de participantes } \\
\text { en las organizaciones y / o } \\
\text { formación de nuevos } \\
\text { actores sociales (formales } \\
\text { o informales) }\end{array}$ & \multirow[t]{2}{*}{$\begin{array}{l}\text { Cambios en la estructura } \\
\text { de costes y beneficios } \\
\text { (previsibles) para la acción } \\
\text { colectiva }\end{array}$} \\
\hline & & $\begin{array}{l}\text { Desenganche (salida de } \\
\text { grupos e individuos) }\end{array}$ & \\
\hline & \multirow[t]{2}{*}{$\begin{array}{l}\text { (2) } \\
\text { Conectividad } \\
\text { social y } \\
\text { networking }\end{array}$} & \multirow[t]{2}{*}{$\begin{array}{l}\text { Variaciones en el número e } \\
\text { intensidad de los contactos } \\
\text { y en la intensidad (más allá } \\
\text { del momento del PP) }\end{array}$} & $\begin{array}{l}\text { La institución } \\
\text { desempeña un rol } \\
\text { facilitador (mecanismo } \\
\text { de intermediación o } \\
\text { brokerage) que vincula } \\
\text { actores divesos y } \\
\text { previamente desconocidos. }\end{array}$ \\
\hline & & & $\begin{array}{l}\text { La institución trae una } \\
\text { selección (políticamente) } \\
\text { sesgada de actores } \\
\text { (mecanismos de cierre) }\end{array}$ \\
\hline \multirow{5}{*}{ 를 } & \multirow[t]{3}{*}{$\begin{array}{l}\text { (3) } \\
\text { Variaciones en } \\
\text { percepciones } \\
\text { mutuas } \\
\text { (entre grupos } \\
\text { sociales) }\end{array}$} & $\begin{array}{l}\text { Escalera de identificación } \\
\text { (primeros peldaños): } \\
\text { Variaciones en } \\
\text { conocimiento }\end{array}$ & \multirow[t]{3}{*}{$\begin{array}{l}\text { Mecanismos de reducción } \\
\text { de incertidumbre } \\
\text { (mediante aumento de } \\
\text { información producto de } \\
\text { la interacción) }\end{array}$} \\
\hline & & $\begin{array}{l}\text { Variaciones en actitudes de } \\
\text { reconocimiento mutuo }\end{array}$ & \\
\hline & & $\begin{array}{l}\text { Variaciones en actitudes de } \\
\text { (des)confianza mutua }\end{array}$ & \\
\hline & \multirow{2}{*}{$\begin{array}{l}\text { (4) } \\
\text { Variaciones en } \\
\text { procesos de } \\
\text { identificación } \\
\text { colectiva }\end{array}$} & $\begin{array}{l}\text { Escalera de identificación } \\
\text { (peldaños superiores): }\end{array}$ & \multirow{2}{*}{$\begin{array}{l}\text { Mecanismos de atribución } \\
\text { de similitudes (y } \\
\text { diferencias) entre actores }\end{array}$} \\
\hline & & $\begin{array}{l}\text { Nuevas identidades } \\
\text { colectivas (supragrupales) }\end{array}$ & \\
\hline
\end{tabular}

Fuente: elaboración propia. 
los PPs no cambiarían la estructura de oportunidades políticas y no estimularían nuevas interacciones entre los grupos asociativos. En algunos otros casos, algunos expertos describen la "salida" de una manera "hirschmaniana», es decir, como una reacción política, o una estrategia de boicot, de actores asociativos para expresar su descontento y resistir procesos de redistribución del poder que son percibidos como desafiantes del statu quo (Ganuza et al., 2014). Por ejemplo, en un caso de presupuesto participativo:

En los primeros años, fue muy difícil trabajar con las asociaciones vecinales, ya que [...] vivían esto como una usurpación de su poder (GN-Profesionales, Andalucía).

Según los expertos, el establecimiento de redes (tabla 1, efecto 2) parece ser uno de los subproductos más relevantes, ya que los PPs reúnen a diferentes organizaciones que inician (o continúan) un proceso de conocimiento mutuo, visualizando e intercambiando información sobre su trabajo y estableciendo relaciones estables de cooperación más allá del proceso. Por ejemplo, un académico (que se mostraba realmente pesimista acerca de los efectos reales de los PPs) explicó cómo un consejo de bienestar, en una ciudad de tamaño mediano, facilitó que las organizaciones comenzaran a coordinar sus agendas:

Las organizaciones sociales tienden a estar mal coordinadas y trabajar por su cuenta. Los consejos participativos favorecen que se reúnan y sirven para coordinar sus acciones, incluso en actividades más allá del ámbito del consejo (entrevista académico).

Los cambios en la red de grupos asociativos también podrían tener expresiones a nivel cultural. Los PPs pueden significar una intensificación de las interacciones (de contactos significativos) entre los actores. Esta sería una nueva oportunidad, en muchos casos, de conocerse entre sí, facilitando la activación de mecanismos de revisión actitudinal (tabla 1, efecto 3) relativa a las percepciones mutuas y a las identificaciones colectivas.

Los participantes tienen la oportunidad de conocerse mutuamente, iniciando procesos de reconocimiento, confianza y, finalmente, identificación como parte de una misma comunidad. Este proceso gradual de cambio de actitud puede ser representado como una «escalera de identificación» (efectos 3 y 4), que también puede transitar hacia abajo, como consecuencia de experiencias negativas ${ }^{15}$. Entre los diversos mecanismos detrás de la formación de

15 Sobre los fundamentos teóricos de estos micromecanismos véase, por ejemplo, el trabajo de Pettigrew (1998) sobre la teoría del contacto intergrupal. A su vez, tal como 
(des)confianza, los PPs pueden activar aquellos basados en un aumento del conocimiento (información), reduciendo la incertidumbre sobre los comportamientos de los demás, o simplemente en el reconocimiento de similitudes (toma de conciencia de compartir, o no, objetivos, valores, tareas, etc. $)^{16}$. Estos procesos también pueden considerarse como micromotores para la formación de identidades colectivas (efecto 4$)^{17}$.

Obviamente, los PPs pueden resultar culturalmente inocuos en ausencia de una interacción duradera, intensa y deliberativa. El mero aumento en las interacciones no garantiza el inicio de procesos de confianza e identificación; y también puede conducir al escenario opuesto (no deseado) de desconfianza social, ausencia de reconocimiento e identificación como adversarios, especialmente cuando los PPs fomentan divisiones o refuerzan desigualdades preexistentes. Por ejemplo, la instrumentalización o apropiación del proceso por parte de determinados actores asociativos puede ser un factor generador de desconfianza, alimentando lógicas de competencia.

Según los expertos, estos efectos culturales parecen inusuales en comparación con los cambios (de tipo estructural) en la red de actores sociales. Sin embargo, algunos expertos, refiriéndose a los presupuestos participativos y otros procesos más duraderos (por ejemplo, los foros permanentes sobre el agua) perciben, como plausibles, algunos de estos cambios en las percepciones mutuas:

En este proceso (foros de agua), que duró cuatro años, se conocen y se reconocen, a pesar de sus desacuerdos. Ahora eran capaces de trabajar juntos. Uno de los participantes dijo que él solía pensar que los ecologistas eran 'hippies sin cerebro', pero que tras pasar muchas horas con ellos, y aunque no se ha convertido en uno de ellos, ahora reconoce sus argumentos, y viceversa (entrevista académico).

se discute más adelante, este tipo de efectos en las percepciones mutuas también pueden conectarse a otro conjunto de efectos (indirectos), como la apertura de la perspectiva (o la mentalidad ampliada). Del mismo modo, un efecto derivado de la combinación de estos cambios de actitud puede ser el establecimiento o refuerzo de patrones de interacción entre grupos basados en la colaboración (como efecto positivo) o en la competencia (cuando este tipo de actitudes está ausente o se desincentiva).

16 Otros mecanismos generadores de confianza sugeridos en las entrevistas son aquellos basados en la reputación, cuando la reputación de los actores principales o las instituciones afectan la revisión de las actitudes.

17 Como se analiza a continuación, también se pueden detectar efectos similares a nivel de la red de política, incluidas las autoridades públicas y otros actores no sociales, como se expone más adelante en el texto. 
En cuanto a las relaciones entre los grupos [en una experiencia de presupuestos participativos], los diferentes grupos a veces dejan de lado su propia identidad para generar una identidad colectiva, que es la del territorio (GN-Profesionales, Andalucía).

\section{H2: LA HIPÓTESIS DEL EMPODERAMIENTO DE LA SOCIEDAD CIVIL: PROCESOS PARTICIPATIVOS COMO FORTALECEDORES DE LA ORGANIZACIÓN SOCIAL}

Sí, de empoderamiento en términos de adquisición de recursos sí, en términos de poder de decisión... menos. La mejora del conocimiento de la Administración, de cómo relacionarse con ella, de recursos, de espacios, sí, claro que sí. Adquieren capacidades o habilidades participativas, yo creo que sí. Igual que se produce a nivel individual, se produce a nivel colectivo (entrevista académico).

En cuanto a las asociaciones, los PPs reducen otras formas de acción colectiva [...] El mero hecho de participar puede desactivar otras luchas de las organizaciones que intentan transformar la realidad social (GN-Profesional, Andalucía).

¿Empoderan los procesos participativos a los grupos asociativos? De acuerdo con la literatura y las evaluaciones de los expertos, el empoderamiento puede ser observado en tres tipos de efectos. Desde un punto de vista estructural, los PPs pueden implicar cambios, en primer lugar, en la cantidad y variedad de recursos organizativos disponibles y pueden, en segundo lugar, favorecer innovaciones en su estilo organizativo (metodologías, agendas, repertorios de acción). En tercer lugar, desde el punto de vista cultural, el empoderamiento se evidenciaría en la aparición de (nuevos) sentimientos de eficacia política entre los actores asociativos.

En primer lugar, la apertura de nuevas oportunidades de participación puede conllevar variaciones en la disponibilidad o el acceso a recursos organizativos (tabla 2, efecto 5), ya sean recursos materiales (como infraestructuras o subsidios públicos), recursos cognitivos (acceso a información, conocimientos administrativos, etc.) o recursos relacionales (por ejemplo, acceso a funcionarios de la Administración y autoridades políticas). Como indica la cita anterior, en opinión de los expertos, los efectos más relevantes parecen ser los relacionadas con los recursos cognitivos y la información, y de manera más específica, sobre cómo funciona la Administración pública. También se activan mecanismos de certificación política, por medio de los cuales las autoridades reconocen a las asociaciones como interlocutores legítimos (McAdam, Tarrow y Tilly, 2001: 145-146). Los PPs otorgan visibilidad pública a los grupos 
asociativos, lo que a su vez puede aumentar su capacidad de movilizar recursos materiales en forma, por ejemplo, de donaciones o voluntariado ${ }^{18}$.

En segundo lugar, los cambios en los recursos disponibles pueden contribuir a desencadenar dinámicas organizativas internas ${ }^{19}$, generalmente asociadas con procesos de consolidación organizativa. Estos pueden ser detectados en cambios en el funcionamiento interno y en la comunicación con sus bases sociales (metodologías), sus objetivos (agendas) y sus actividades externas (repertorio político) (efecto 6). Este tipo de efecto es mucho más difícil de entender y operacionalizar ya que se refiere principalmente a las tendencias organizativas a largo plazo. Sin embargo, en las entrevistas de los expertos, es recurrente que se señalen las consecuencias negativas que subyacen.

En el centro de estos discursos podemos pensar en la activación de dos mecanismos diferentes, aunque interrelacionados. Por un lado, el acceso a los espacios institucionales puede activar procesos de institucionalización, mediante los cuales las organizaciones se asimilan a las normas institucionales (adoptando puntos de vista y formas de acción dominantes). Los expertos tienden a conectar estas tendencias con los procesos de desmovilización política o desradicalización, cuando no de cooptación. Como en la cita anterior, los PPs pueden desviar las energías limitadas de las organizaciones hacia objetivos institucionales menos transformadores o desafiantes.

Además, los PPs pueden favorecer la activación de un mecanismo de burocratización y moderación ideológica. Aquí la participación institucionalizada estimula ciertos patrones de desarrollo organizativo caracterizados por la toma de decisiones jerárquica, el debilitamiento de los lazos con las bases sociales y el desplazamiento de las demandas políticas en favor de los objetivos de supervivencia de la propia organización. Aunque el recurso a esta narrativa es común entre los expertos, en algunas ocasiones también destacan los casos en que los PPs (los presupuestos participativos específicamente) llevaron a las asociaciones a abrirse hacia sus comunidades de referencia. Del mismo modo, los profesionales se refieren a los PPs como estimulantes de democracia interna por la difusión de herramientas para el debate y la toma de decisiones con metodologías participativas y deliberativas (igualdad de participación, respeto, igual consideración de argumentos, etc.). Por lo tanto, los PPs también pueden activar mecanismos de desburocratización en las organizaciones.

18 La visibilidad pública desempeña un papel crucial en la ampliación del potencial de movilización de las organizaciones y movimientos sociales, ya sea en forma de trabajo voluntario o participantes en protestas (Klandermans y Oegema, 1989).

19 Por lo tanto, este conjunto de efectos puede entenderse como una consecuencias indirecta, en gran medida dependiente de anterior (las variaciones en el acceso de los actores sociales a los recursos). 
En consecuencia, desde un punto de vista estructural, los PPs no solo pueden proporcionar a los grupos asociativos nuevos recursos (empoderando), sino que también pueden transformar los repertorios de acción, las metodologías y agendas. En ocasiones, estos cambios pueden suponer la moderación de las demandas más desafiantes (desempoderando). Una contradicción similar puede verse cuando examinamos los cambios culturales dentro de los grupos.

Tabla 2. La hipótesis del empoderamiento de la sociedad civil: procesos participativos como fortalecedores de la organización social

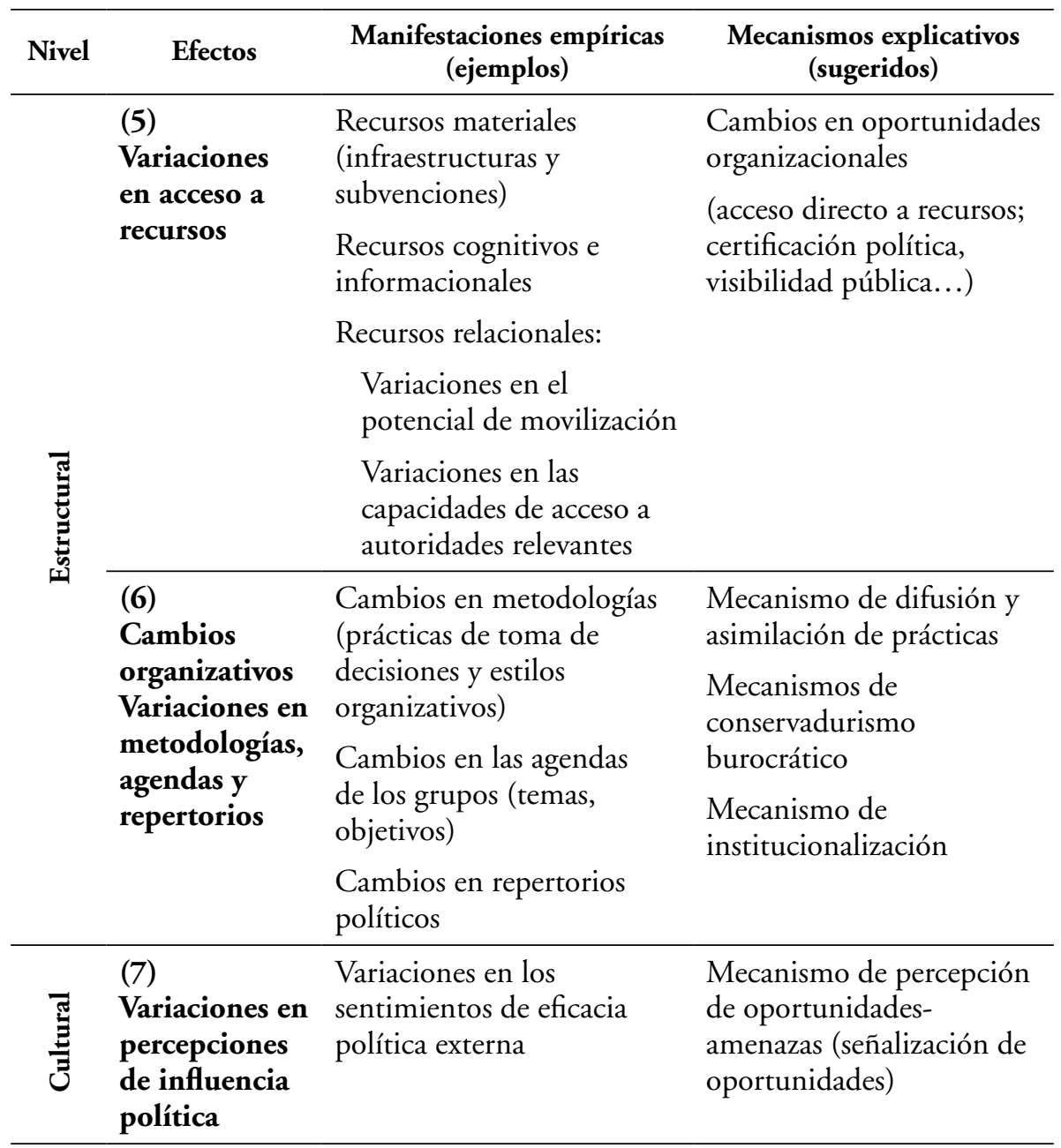

Fuente: elaboración propia. 
A nivel cultural, los PPs pueden incrementar el empoderamiento a través del fortalecimiento de sentimientos de eficacia externa o influencia política. Este efecto está vinculado a la percepción de las oportunidades que conduce a juicios favorables sobre la capacidad de influir y lograr cambios en las políticas (efecto 7). La evaluación de los participantes sobre su influencia (utilizando, por ejemplo, los recuerdos sobre el grado de cumplimiento de las decisiones) puede conducir a un mayor compromiso con el propio proceso participativo y puede contribuir también a revisar las actitudes hacia las autoridades públicas. Por el contrario, los PPs pueden generar frustración y disminuir la percepción de eficacia política, alentando procesos de salida, desconfianza y rechazo de futuros procesos institucionales.

En la literatura de los movimientos sociales, el aumento de la eficacia externa es un proceso clave en los procesos de movilización (Piven y Cloward, 1979; McAdam, 1982). Del mismo modo, los PPs pueden generar sentimientos de eficacia y multiplicar la movilización. Sin embargo, los expertos entrevistados consideran que rara vez ocurre en términos positivos. En el medio plazo, la ausencia de influencia política termina por generar frustración y una percepción de los PPs como una pérdida de tiempo. Como señala un académico:

Generalmente, cuando lo que se acuerda no se cumple, la gente ve que es poco útil [...] Se habla mucho, se debate mucho pero no se llegan a acuerdos. O el que gobierna no los cumple [...] Se genera desmotivación. Se crea una memoria colectiva negativa (entrevista académico).

Si se espera que los PPs produzcan empoderamiento en las asociaciones y que aumenten los sentimientos de eficacia política ("podemos tener voz»), según nuestros expertos, el efecto contrario (frustración de expectativas y sentimientos de ineficacia política externa) resulta frecuente. Por lo tanto, los PPs que estaban destinados a empoderar a la sociedad, a veces, generan frustración y desempoderan.

\section{H3. LA HIPÓTESIS DEL CAMBIO EN LAS RELACIONES DE PODER: EL PROCESO PARTICIPATIVO COMO TRANSFORMADOR DE LOS PATRONES DE INTERACCIÓN DOMINANTES}

¿Democratizan los procesos participativos los patrones de interacción entre los grupos asociativos y las autoridades? Una preocupación central en las justificaciones normativas de los PPs es la transformación de la estructura de poder de la sociedad civil y de las autoridades y su lógica de interacción. Para comprender empíricamente tal cambio en las relaciones de poder, nos 
centramos en las redes de políticas (policy networks) y en los procesos de toma de decisiones después de los PPs. Siguiendo el enfoque relacional propuesto por Baiocchi et al. $(2008 ; 2011)$, nuestra propuesta agrupa seis efectos diferentes, aunque interrelacionado ${ }^{20}$. El primer efecto se refiere a las variaciones en el número de actores involucrados en las redes de política como consecuencia de las nuevas oportunidades (o barreras) de acceso derivadass PPs (tabla 3, efecto 8). Este efecto aparece relacionado con el efecto 1 (en la tabla 1), sin embargo aquí el acento se pone en los cambios en el conjunto de la red de la política (incluyendo autoridades políticas y funcionarios y personal de la Administración). En este sentido, los PPs pueden conducir a la incorporación o mantenimiento de nuevos actores asociativos previamente excluidos (Hernández-Medina, 2010; Wampler, 2007). Pero, como ya se ha mencionado, los PPs también pueden activar mecanismos de cierre, reduciendo el número de voces que acceden a la red de políticas o ubicándolas en posiciones marginales (Aguiar y Navarro, 2000). Como planteaba uno de los académicos entrevistados:

El modelo clientelista, si se quiere, el de la selección de los participantes, no ha desaparecido. De hecho, hay muchas organizaciones sociales que piensan que debe seguir siendo así (entrevista académico).

Los tres efectos subsiguientes en la tabla 3 son expresiones del potencial transformador de los PPs en tres propiedades de la red de políticas: la transparencia en las interacciones — como opuesta a la política opaca (efecto 9)—; los cambios en los patrones de autoridad (efecto 10) y la prevalencia de dinámicas de colaboración —en lugar de conflictivas (efecto 11).

Un efecto clave de los PPs se relaciona con la institucionalización de prácticas de transparencia. Cuando las nuevas reglas son ampliamente respaldadas por la (nueva) constelación de actores, podrían institucionalizarse nuevos principios de interacción, como los relacionados con la transparencia (en oposición a la opacidad) (efecto 9). Por ejemplo, los cambios en los flujos de información, provocados por la formalización y publicidad de las interacciones, pueden reducir el ejercicio del poder discrecional por parte de las autoridades y actores asociativos, y pueden aumentar la capacidad de influencia de quienes carecen de poder. Un segundo indicador de transparencia se manifiesta en el grado de publicidad o divulgación de los eventos políticos. Así, tras la implementación de PPs, las prácticas de transparencia pueden

20 En otro lugar hemos desarrollado y aplicado empíricamente esta propuesta (Behrer et al., 2016). 
extenderse a otras esferas, aumentando así la información disponible sobre otros procedimientos. Por ejemplo, puede haber más información sobre la adopción, el rechazo o el momento de la implementación de las propuestas. Esto es crítico para el monitoreo o control público de la toma de decisiones (Bauhr y Grimes, 2014).

De acuerdo con la literatura, los PPs también pueden impulsar procesos de redistribución del poder entre los actores, proporcionando nuevas oportunidades políticas para influir en las decisiones (Fagotto y Fung, 2006, 2009). Por ejemplo, un PP desencadena un proceso de toma de decisiones compartida en un área de política específica que, en el futuro, puede ser replicado en otras áreas. Este tercer efecto es crucial para entender cualquier proceso de transformación en los patrones de interacción establecidos, desde aquellos basados en el clientelismo hacia modos de interacción más transparentes (Abers, 1998; Montambeault, 2011).

Los cambios de poder provocados por los PPs pueden mejorar la capacidad de los actores asociativos para promover sus propios intereses y metas en entornos de toma de decisiones relevantes (Baiocchi et al., 2011). Sin embargo, también pueden conducir a, o reforzar, la cooptación política (Selznick, 1948). El clientelismo y la cooptación a nivel local pueden ser vistos como una forma de "pork-barrel politics» en la asignación de recursos públicos (Ferejohn, 1974), no necesariamente asociada a la corrupción. Bajo este modo de interacción, las organizaciones sociales pueden ser internamente autónomas pero, en su interacción con las autoridades, depende de relaciones clientelares (Baiocchi et al., 2008). Esto ocurre cuando la interacción implica la alineación y complicidad de los grupos asociativos, sus intereses y objetivos con respecto a los grupos con más poder o hegemónicos — normalmente, el partido político en el gobierno- . De manera similar, algunos actores - habitualmente, las autoridades públicas- pueden utilizar los procesos de participación para sus propios fines (Baker, 1999). En este sentido, los PPs pueden servir para reforzar (u ocultar) los patrones clientelares preexistentes.

Otro efecto potencial (efecto 10) está relacionado con el grado de conflicto y cooperación en la interacción. Los PPs pueden traer la cooperación a contextos conflictivos previos o, a la inversa, pueden conducir a escenarios de conflicto cuando los actores establecidos - ya sean autoridades públicas o actores asociativos - resisten o boicotean la institucionalización de nuevas prácticas transparentes (Ganuza et al., 2014). No obstante, aumentos momentáneos en el nivel de conflicto pueden señalar la activación de la redistribución de poder, que, si no se abordan, pueden conducir a un fracaso de la institución. 
Tabla 3. La hipótesis del cambio en las relaciones de poder: el proceso participativo como transformador de los patrones de interacción sociedad civil-autoridades

\begin{tabular}{|c|c|c|c|}
\hline Nivel & Efectos & $\begin{array}{c}\text { Manifestaciones empíricas } \\
\text { (ejemplos) }\end{array}$ & $\begin{array}{c}\text { Mecanismos explicativos } \\
\text { (sugeridos) }\end{array}$ \\
\hline 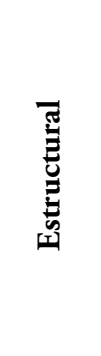 & $\begin{array}{l}\text { (8) } \\
\text { Selectividad de } \\
\text { la policy arena } \\
\text { (pluralismo) }\end{array}$ & $\begin{array}{l}\text { Inclusividad de la policy } \\
\text { arena (y diversidad de } \\
\text { actores) }\end{array}$ & $\begin{array}{l}\text { Mecanismos de cambio } \\
\text { en las oportunidades } \\
\text { políticas: } \\
\text { - Aperturas-cierres } \\
\text { estructurales } \\
\text { - Percepciones de } \\
\text { oportunidades } \\
\text { significativas }\end{array}$ \\
\hline 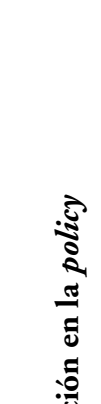 & $\begin{array}{l}\text { (9) } \\
\text { Transparencia } \\
\text { vs. opacidad }\end{array}$ & $\begin{array}{l}\text { Variaciones en pautas } \\
\text { de comunicación e } \\
\text { interacción } \\
\text { Variaciones en el grado de } \\
\text { publicitación de los policy } \\
\text { events }\end{array}$ & $\begin{array}{l}\text { Mecanismos de } \\
\text { institucionalización de la } \\
\text { policy arena: } \\
\text { - Internalización de } \\
\text { (nuevos) principios y } \\
\text { prácticas democráticas } \\
\text { como guías de la } \\
\text { interacción y los flujos } \\
\text { de información }\end{array}$ \\
\hline 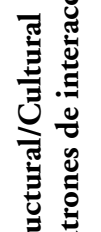 & $\begin{array}{l}\text { (10) } \\
\text { Autoridad }\end{array}$ & $\begin{array}{l}\text { Aumentos y pérdidas en } \\
\text { autonomía de los actores } \\
\text { sociales }\end{array}$ & $\begin{array}{l}\text { Mecanismos de cambio } \\
\text { de las oportunidades } \\
\text { políticas: } \\
\text { - Redistribución } \\
\text { (estructural) de poder }\end{array}$ \\
\hline 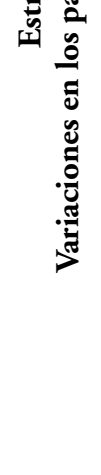 & $\begin{array}{l}(11) \\
\text { Cooperación } \\
\text { vs. conflicto }\end{array}$ & $\begin{array}{l}\text { Variaciones en las pautas } \\
\text { de cooperación y conflicto }\end{array}$ & $\begin{array}{l}\text { Mecanismos de cambio de } \\
\text { oportunidades políticas: } \\
\text { - Percepción de } \\
\text { oportunidades/ amenazas } \\
\text { Mecanismos de } \\
\text { institucionalización de la } \\
\text { policy arena: } \\
\text { - Compromisos } \\
\text { normativos (nuevas } \\
\text { normas) }\end{array}$ \\
\hline
\end{tabular}




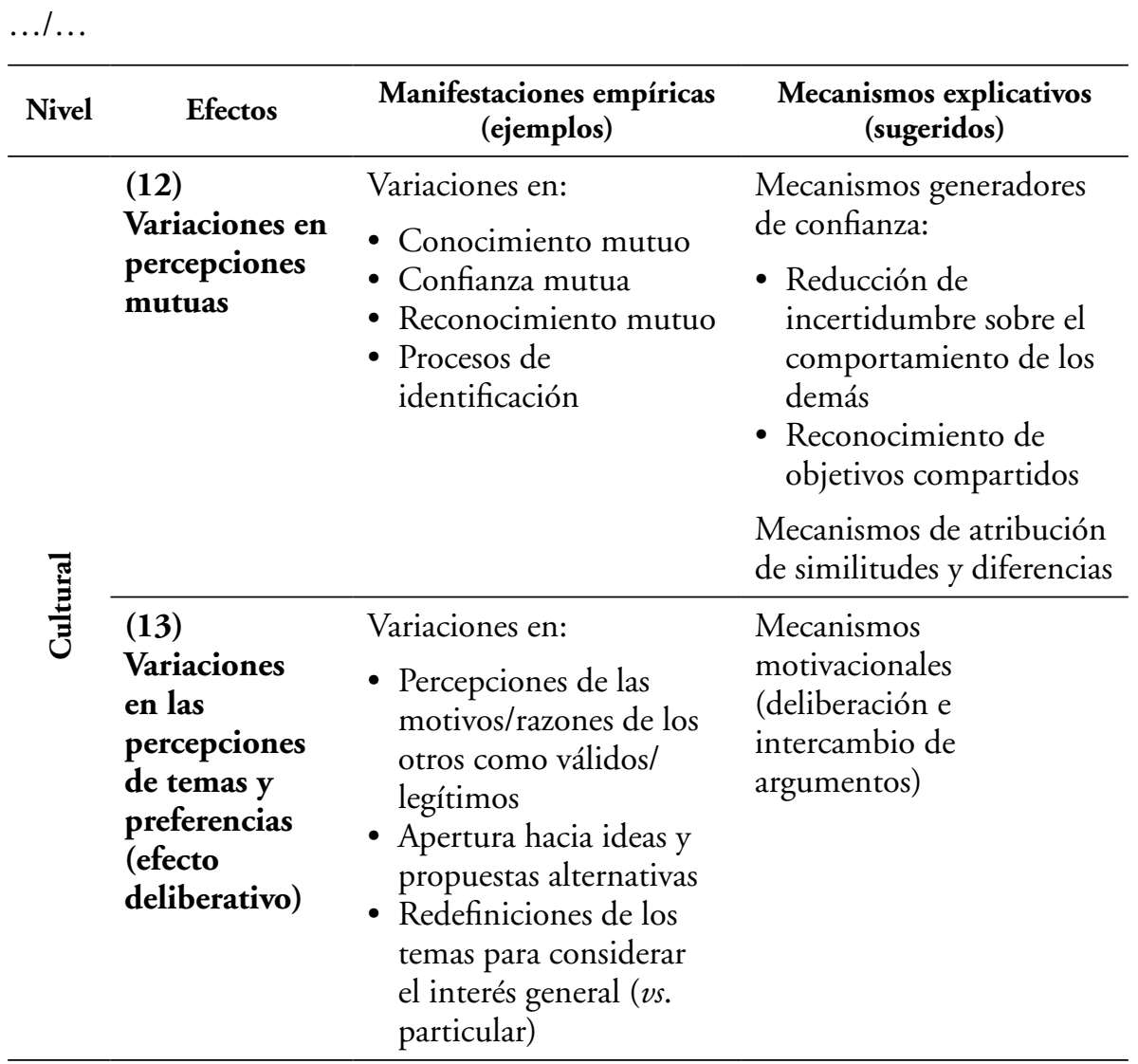

Fuente: elaboración propia.

Según los discursos de los expertos, estos cambios estructurales (apertura de las redes de política y aumento de las interacciones significativas) ocurren, en ocasiones, en los procesos más intensos como los presupuestos participativos o los planes estratégicos. Estos procesos abren espacios para grupos previamente excluidos. Esta «apertura» de la red de políticas se manifiesta, por ejemplo, en el establecimiento por norma de la "obligación» de hacer convocatorias abiertas antes de cualquier decisión relevante:

[Los PPs] han hecho asumir que como mínimo debe consultarse a la ciudadanía (GN profesionales-Barcelona).

Seis años después del proceso, hubo una parte sustantiva de los ingenieros, técnicos... que dijeron algo como que ya no se imaginaban hacer política, por ejemplo, aprobar un plan de gestión, sin participación, sin diálogo, no se puede 
encerrar en el despacho por muy técnico que sea. En la Administración sí ha habido cambio (entrevista académico).

Sin embargo, parece que a pesar de la extensión de los PPs, la consolidación de nuevos patrones de interacción como consecuencia de la redistribución del poder parece poco probable. Según los expertos, los PPs se asocian más comúnmente con la inocuidad política o con la reproducción de modos clientelares de interacción. En todos los casos, directa o indirectamente, intencionalmente o no, a largo plazo los PPs parecen estar más cerca de integrarse en la forma habitual de hacer política que de volverla del revés.

En los procesos participativos, aun no queriendo, se siguen reproduciendo situaciones clientelares [...] participan los mismos [...] Al final, se acaba haciendo participación entre comillas, más o menos, para ciertos grupos (GN profesionales-Cataluña).

Los cambios estructurales en las relaciones de poder pueden igualmente expresarse a nivel cultural. Los cambios se resumen aquí en dos tipos de efectos. Por un lado, las variaciones en las percepciones mutuas (efecto 12) entre los miembros de la red de política (particularmente con las autoridades políticas). Por otro lado, los cambios en la forma en que se definen las cuestiones y se consideran las preferencias (el efecto deliberativo).

De la misma manera que los efectos 3 y 4 (en la tabla 1 ) se refieren a los cambios en las actitudes de los grupos asociativos (la «escalera de identificación»), podemos clasificar diversos cambios en las actitudes de percepción mutua entre asociaciones y autoridades públicas. Se trataría de un proceso gradual de «integración actitudinal» entre los participantes en la red de políticas (efecto 12). Cuando los PPs alientan una comunicación intensa y políticamente significativa, cabe esperar un incremento en el conocimiento mutuo, activando, a su vez, mecanismos de generación de confianza: reducción de la incertidumbre sobre los comportamientos de los demás, reconocimiento de objetivos compartidos, etc. Y, finalmente, pueden fomentar la identificación mutua (atribución de similitudes) como miembros de una red de política. Obviamente, los cambios en las percepciones mutuas pueden seguir un patrón negativo, por ejemplo, cuando los actores asociativos son excluidos (formal o informalmente) o sus expectativas no se cumplen. En estos casos, los PPs pueden favorecer actitudes de rechazo mutuo, desconfianza, falta de reconocimiento, etc.

Del mismo modo, y vinculado a mecanismos previos de confianza y reconocimiento, la comunicación intensa y significativa puede conducir a cambios en la percepción de los problemas y las preferencias de los actores 
(efecto 13). Los cambios (positivos) en las percepciones mutuas pueden conducir, a su vez, a una mayor receptividad a las nuevas ideas y a la toma de perspectiva o empatía (entendiendo las opiniones y necesidades de los demás). Además, según los enfoques de la democracia deliberativa, como resultado de la interacción comunicativa, podemos esperar la activación de mecanismos deliberativos (o el «efecto motivacional» de Cohen) que favorecen la adopción de orientaciones hacia el bien común (o intereses generales) frente a posiciones basadas en intereses egoístas (véanse Cohen, 1989, o Pincock, 2012).

En opinión de nuestros expertos, estos efectos culturales suceden a veces; pero ponen especial énfasis en los efectos relativos al conocimiento mutuo y el reconocimiento de los roles y prácticas de los demás. Para ellos, los cambios normalmente se limitan a los peldaños inferiores:

[Si ha habido un incremento en la] comprensión de la postura de los organismos y funciones de los diferentes agentes, es decir, la gente conoce cómo funciona el ayuntamiento e, incluso, [en el tema de] los presupuestos, con el tiempo aprendes a saber qué puedes solicitar y qué no [...] Pero también el ayuntamiento o en las administraciones reconocen los procesos internos que se dan en las organizaciones, y cómo y porqué se toman las decisiones (GN- profesionales, Andalucía).

[En el caso de una política sectorial] [Los actores] decían que lo más importante y permanente en el tiempo fue el hecho de haber conocido a otros. $Y$ aunque siguen siendo adversarios políticos, ahora reconocen sus argumentos y posiciones... (entrevista académico).

El conocimiento y reconocimiento mutuos pueden adaptarse también al clientelismo y a la instrumentalización. Según los expertos, los PPs reproducen patrones de interacción que normalmente implican la «sumisión mutua»: las autoridades locales adquieren el papel de «donantes» acríticos y las organizaciones sociales juegan el papel de demandantes no desafiantes.

\section{CONCLUSIONES}

Desde la perspectiva de la democracia y la sociedad civil, no podemos afirmar que los procesos participativos no tengan efectos. Pueden ser los potenciadores democráticos imaginados desde los planteamientos normativos (Cohen y Rogers, 1995), o pueden servir como sustentadores del statu quo. Incluso cuando pueden resultar aparentemente inocuos, tienen efectos, ya que con frecuencia pueden conducir a la frustración participativa (Fernández et 
al., 2016). En definitiva, los procesos participativos pueden generar múltiples efectos (potenciales) que pueden influir (positiva o negativamente) en la calidad democrática de nuestro sistema político. En este trabajo hemos explorado el bosque de efectos potenciales en un esfuerzo por articularlos en un marco analítico para su análisis empírico.

Además de la revisión bibliográfica, nos hemos apoyado en las opiniones de expertos, especialistas y profesionales de la participación pública. Funcionando como orientadores, los discursos de los expertos nos han guiado fructíferamente en la articulación de un marco analítico. La propuesta resultante organiza una serie de efectos identificados, negativos y positivos, en torno a tres hipótesis o ideas generales sobre los cambios democratizadores que pueden generar los procesos participativos: el aumento del capital social, la potenciación de los grupos sociales y la democratización de la interacción de la sociedad civil y las autoridades políticas. La propuesta también implica un esfuerzo para proporcionar ejemplos de manifestaciones empíricas de estos efectos y para especificar los mecanismos explicativos asociados a los mismos.

En general, este trabajo ayudará a investigadores, académicos y profesionales a pensar en términos de qué efectos, mecanismos y evidencias deben ser considerados en los estudios que abordan las consecuencias democratizadoras de los procesos participativos en la sociedad civil y su interacción con el Estado. Esperamos contribuir a mejorar la calidad (y la comparabilidad) de los trabajos empíricos futuros así como al planteamiento de evaluaciones de resultados en este terreno.

Pensamos que resultará aplicable en una amplia variedad de experiencias participativas. Sin embargo, nuestra propuesta tiene también limitaciones. En este sentido, se deben considerar tres observaciones. En primer lugar, hay que tener en cuenta que la mayoría de los efectos, especialmente aquellos vinculados a la mejora democrática, solo se esperan en los casos de mecanismos participativos que suponen interacciones regulares (duraderas en el tiempo) intensas y políticamente significativas entre actores heterogéneos (Fung, 2006). Como algunos expertos entrevistados indicaron, la discontinuidad en la implementación de los PPs solo nos permite observar su potencialidad transformadora en efectos incipientes. En segundo lugar, no debemos olvidar que el conocimiento de nuestros expertos sobre procesos participativos está circunscrito en gran medida al contexto español y se refiere a un número reducido de mecanismos, en su mayoría consejos consultivos y presupuestos participativos. En este sentido, la propuesta analítica será de mayor interés para los interesados en las consecuencias de los procesos participativos en España. No obstante, pensamos que también ofrece muchas ideas que son extrapolables a otros contextos. Por último, en relación con las manifestaciones empíricas o los indicadores propuestos, debe tenerse en cuenta que se proporcionan como 
ejemplos, en su mayoría pensando en una investigación hipotética basada en entrevistas a los propios participantes y el análisis de documentos como principales procedimientos para obtener evidencia empírica.

\section{Bibliografía}

Abers, R. (1998). From clientelism to cooperation: Local government, participatory policy and civic organizing in Porto Alegre, Brazil. Politics and Society, 26 (4), 511-537. Disponible en: https://doi.org/10.1177/0032329298026004004.

_ (2003). Reflections on what makes empowered participatory governance happen. En A. Fung, E. Olin Wright y R. Abers (eds.). Deepening democracy: Institutional innovations in empowered participatory governance (pp. 200-208). New York: Verso.

(2007). Organizing for governance: building collaboration in Brazilian river basins. World Development, 35 (8), 1450-1463. Disponible en: https://doi.org/10.1016/j. worlddev.2007.04.008.

Aguiar, F. y Navarro, C. J. (2000). Democracia y participación ciudadana en los municipios: ¿un mercado político de trastos? Revista Española de Investigaciones Sociológicas, 91, 89-111.

Alberich, T., Arnanz, L., Basagoiti, M., Belmonte, R., Bru, P., Espinar, C. y Lorenzana, C. (2009). Metodologias participativas. Madrid: CIMAS.

Bachrach, P. y Baratz, M. S. (1962). Two faces of power. American Political Science Review, 56 (4), 947-952. Disponible en: https://doi.org/ 10.1017/S0003055406222561.

Baiocchi, G., Heller, P., y Silva, M. K. (2008). Making space for civil society: Institutional reforms and local democracy in Brazil. Social Forces, 86 (3), 911-936. Disponible en: https://doi.org/10.1353/sof.0.0015.

— y Silva, M. (2011). Bootstrapping democracy: Transforming local governance and civil society in Brazil. Stanford, CA: Stanford University Press.

Baker, G. (1999). The taming of the idea of civil society. Democratization, 6 (3), 1-29. Disponible en: https://doi.org/10.1080/13510349908403619.

Barber, B. (2003). Strong democracy: Participatory politics for a new age. Berkeley, Los Angeles: University of California Press.

Bauhr, M. y Grimes, M. (2014). Indignation or resignation: the implications of transparency for societal accountability. Governance, 27 (2), 291-320.

Bergman, M. M. y Coxon, A. P. (2005). The quality in qualitative methods. En Forum Qualitative Sozialforschung/Forum: Qualitative Social Research, 6 (2), art. 34. Disponible en: http://www.qualitative-research.net/index.php/fqs/article/view/457/975.

Bherer, L., Fernández-Martínez, J. L., García Espín, P. y Jiménez Sánchez, M. (2016). The promise for democratic deepening: the effects of participatory processes in the interaction between civil society and local governments. Journal of Civil Society, 12 (3), 344363. Disponible en: https://doi.org/10.1080/17448689.2016.1215957.

Bherer, L., Gauthier, M. y Simard, S. (2016). Introduction. En L. Bherer, M. Gauthier y S. Simard (eds.). The Professionalization of Public Participation. New York-London: Taylor and Francis. 
Blanco, I. y Ballester, M. (2011). ¿Participar para transformar? La experiencia de los presupuestos participativos en la provincia de Barcelona. Gestión y Análisis de Políticas Públicas, 5, 117-144. Disponible en: https://doi.org/10.24965/gapp.v0i5.448.

Boyatzis, R. E. (1998). Transforming qualitative information: Thematic analysis and code development. London: Sage.

Cohen, J. (1989). Deliberation and Democratic Legitimacy. En A. Hamlin y P. Pettit (eds.). The Good Polity: Normative analysis of the state (pp. 17-34). New York: Basil Blackwell.

- y Arato, A. (1992). Civil society and political theory. Cambridge: MIT Press.

- y Rogers, J. (1995). Associations and democracy (vol. 15). London: Verso.

Dryzek, J. S. (2000). Deliberative democracy and beyond: liberals, critics, contestations. New York: Oxford University Press.

Eliasoph, N. (1998). Avoiding politics: How Americans produce apathy in everyday life. Cambridge: Cambridge University Press. Disponible en: https://doi.org/10.1017/ CBO9780511583391.

Evans, P. (1996). Government action, social capital and development: Reviewing the evidence on synergy. World development, 24 (6), 1119-1132. Disponible en: https://doi. org/10.1016/0305-750X(96)00021-6.

Fagotto, E. y Fung, A. (2006). Empowered participation in urban governance: the Minneapolis neighborhood revitalization program. International Journal of Urban and Regional Research, 30 (3), 638-655. Disponible en: https://doi.org/ 10.1111/j.1468-2427.2006.00685.x.

- (2009). Sustaining public engagement: Embedded deliberation in local communities. East Hartford, CT: Everyday Democracy and Kettering Foundation. Disponible en: http:// icma.org/en/icma/home.

Ferejohn, J. A. (1974). Pork barrel politics: Rivers and harbors legislation, 1947-1968. Stanford: Stanford University Press.

Fernández Martínez, J., García Espín, P. y Jiménez Sánchez, M. (2016). La frustración participativa como legado cultural de los procesos participativos locales, paper presented at the XII Congreso Español de Sociología, Gijón, 30 junio-2 julio.

Fishkin, J. (1997). The voice of the people: Public opinion and democracy. New Heaven: Yale University Press.

Font, J. y Alarcón, P. (2014). Where are the boundaries of deliberation and participation? A transatlantic debate. Journal of Public Deliberation, 10 (2), art. 11. Disponible en: http://www.publicdeliberation.net/jpd/vol10/iss2/art11.

Font, J., Della Porta, D. y Sintomer, Y. (2014). Participatory democracy in Southern Europe: causes, characteristics and consequences. London: Rowman and Littlefield International.

Funes, M. J., Talpin, J. y Rull, M. (2014). The cultural consequences of engagement in democratic processes. En J. Font, D. della Porta y Y. Sintomer. Participatory democracy in Southern Europe: causes, characteristics and consequences (pp. 151-190). London: Rowman and Littlefield International.

Fung, A. (2003). Survey article: Recipes for public spheres: Eight institutional design choices and their consequences. The Journal of Political Philosophy, 11 (3), 338-367. Disponible en: https://doi.org doi/10.1111/1467-9760.00181.

(2006). Varieties of participation in complex governance. Public Administration Review, 66 (1), 66-75. Disponible en: https://doi.org/ 10.1111/j.1540-6210.2006.00667.x. 
- (2009). Empowered participation: Reinventing urban democracy. Princeton-Oxford: Princeton University Press. Disponible en: https://doi.org/10.1515/9781400835638.

- y Wright, E. O. (2001). Deepening democracy: Innovations in empowered participatory governance. Politics and Society, 29 (1), 5-42. Disponible en: https://doi.org/10.1177/0 032329201029001002.

— (2003). Thinking about empowered participatory governance. En A. Fung y E. O. Wright (eds.). Deepening democracy: Institutional innovations in empowered participatory governance (pp. 3-42). London: Verso.

Ganuza, E. y Baiocchi, G. (2012). The power of ambiguity: How participatory budgeting travels the globe. Journal of Public Deliberation, 8 (2), art.8. Disponible en: http://www. publicdeliberation.net/jpd/vol8/iss2/art8.

Ganuza, E. y Francés, F. (2012). El círculo virtuoso de la democracia: los presupuestos participativos a debate. Madrid: Centro de Investigaciones Sociológicas.

Ganuza, E., Nez, H. y Morales, E. (2014). The struggle for a voice: Tensions between associations and citizens in participatory budgeting. International Journal of Urban and Regional Research, 38 (6), 2274-2291. Disponible en: https://doi.org/ 10.1111/1468-2427.12059.

Gaventa, J. (2006). Triumph, deficit or contestation: Deepening the 'deepening democracy' debate', IDS Working Paper, 264. Brighton: Institute of Development Studies. Disponible en: http://www.ids.ac.uk/publications/ids-series-titles/ids-working-papers.

Hall, P. A. (1986). Governing the economy: The politics of state intervention in Britain and France. New York: Oxford University Press.

Hartz-Karp, J. (2007). How and why deliberative democracy enables co-intelligence and brings wisdom to governance. Journal of Public Deliberation, 3 (1), art.11. Disponible en: http://www.publicdeliberation.net/jpd/vol3/iss1/art6.

Heinelt, H. (2007). Do policies determine politics? En F. Fischer, G. Miller y M. Sidney (ed.). Handbook of public policy analysis. Theory, politics, and methods (pp. 109-119). Boca Ratón-London-New York: CRC Press.

Hernández-Medina, E. (2010). Social inclusion through participation: The case of the participatory budget in São Paulo. International Journal of Urban and Regional Research, 34 (3), 512-532. Disponible en: https://doi.org/10.1111/j.1468-2427.2010.00966.x.

Hirschman, A. (1982). Exit, voice and loyalty. Responses to decline in firms, organizations, and states. Cambridge: Harvard University Press.

Jackman, R. W. y Miller, R. A. (1998). Social capital and politics. Annual Review of Political Science, 1 (1), 47-73. Disponible en: https://doi.org/ 10.1146/annurev.polisci.1.1.47.

Jiménez Sánchez, M. (2005). El impacto politico de los movimientos sociales: un estudio de la protesta ambiental en España. Madrid: CIS.

Kathi, P. C. y Cooper, T. L. (2005). Democratizing the administrative state: Connecting neighborhood councils and city agencies. Public Administration Review, 65 (5), 559567. Disponible en: https://doi.org/10.1111/j.1540-6210.2005.00483.x.

Kinney, B. I. (2012). Deliberation's Contribution to Community Capacity-Building. En T. Nabatchi, J. Gastil, G. M. Weiksner y M. Leighninger (eds.). Democracy in motion: Evaluating the practice and impact of deliberative civic engagement (pp. 163-169). Oxford: Oxford University Press. Disponible en: https://doi.org/10.1093/acprof:oso/9780199899265.003.0008. 
Klandermans, B. y Oegema, D. (1989). Potentials, networks, motivations, and barriers: Steps towards participation in social movements. American Sociological Review, 52 (4), 519531. Disponible en: https://doi.org/10.2307/2095297.

Lowi, T. J. (1972). Four systems of policy, politics, and choice. Public Administration Review, 32 (4), 298-310. Disponible en: https://doi.org/10.2307/974990.

McAdam, D. (1982). Political process and the development of the black insurgency 1930-1970. Chicago: The University of Chicago Press.

— Tarrow, S. y Tilly, C. (2001). Dynamics of contention. Cambridge: Cambridge University Press. Disponible en: https://doi.org/10.1017/CBO9780511805431.

Montambeault, F. (2011). Overcoming clientelism through local participatory institutions in Mexico: What type of participation? Latin American Politics and Society, 53 (1), 91-124. Disponible en: https://doi.org/10.1111/j.1548-2456.2011.00110.x.

Nabatchi, T., Gastil, J., Weiksner, G. M. y Leighninger, M. (eds.) (2012). Democracy in motion: Evaluating the practice and impact of deliberative civic engagement. Oxford: Oxford University Press. Disponible en: https://doi.org/10.1093/acprof:oso/9780199899265.001.0001.

Navarro, C. J. (1999). El sesgo participativo. Madrid: CSIC.

Parkinson, J. (2004). Why deliberate? The encounter between deliberation and new public managers. Public Administration, 82 (2), 377-395. Disponible en: https://doi. org/10.1111/j.0033-3298.2004.00399.x.

Pateman, C. (1970). Participation and democratic theory. Cambridge: Cambridge UP. Disponible en: https://doi.org/10.1017/CBO9780511720444.

Pettigrew, T. F. (1998). Intergroup contact theory. Annual Review of Psychology, 49 (1), 65-85. Disponible en: https://doi.org/10.1146/annurev.psych.49.1.65.

Pincock, H. (2012). Does deliberation make better citizens? En T. Nabatchi, J. Gastil, G. M. Weiksner y M. Leighninger (eds.). Democracy in motion: Evaluating the practice and impact of deliberative civic engagement (pp. 135-162). New York: Oxford University Press. Disponible en: https://doi.org/10.1093/acprof:oso/9780199899265.003.0007.

Piven, F. F. y Cloward, R. A. (1979). Poor people's movements: Why they succeed, how they fail. New York: Vintage Books.

Putnam, R. D., Leonardi, R. y Nanetti, R. Y. (1994). Making democracy work: Civic traditions in modern Italy. Princeton: Princeton University Press.

Selznick, P. (1948). Foundations of the Theory of Organization. American Sociological Review, 13 (1), 25-35. Disponible en: https://doi.org/10.2307/2086752.

Sieber, S. D. (1973). The integration of fieldwork and survey methods. American Journal of Sociology, 78 (6), 1335-1359. Disponible en: https://doi.org/10.1086/225467.

Sintomer, Y. y Ganuza, E. (2011). Democracia participativa y modernización de los servicios públicos: Una investigación sobre las experiencias de presupuestos participativos en Europa. Ámsterdam: Paris TNI/La Découverte.

Smith, G. (2009). Democratic innovations: Designing institutions for citizen participation. Cambridge: Cambridge University Press. Disponible en: https://doi.org/10.1017/ CBO9780511609848.

Stebbins, R. A. (2001). Exploratory research in the social sciences. London: Sage. Disponible en: https://doi.org/10.4135/9781412984249. 
Talpin, J. (2011). Schools of democracy. How (sometimes) ordinary citizens become competent in participatory budgeting institutions. Essex: ECPR Press.

Tarrow, S. (1996). Making social science work across space and time: A critical reflection on Robert Putnam's Making Democracy Work. American Political Science Review, 90 (2), 389-397. Disponible en: https://doi.org/10.2307/2082892.

- (2001). Transnational politics: Contention and institutions in international politics. Annual Review of Political Science, 4, 1-20. Disponible en: https://doi.org/10.1146/ annurev.polisci.4.1.1.

Thelen, K. (1999). Historical institutionalism in comparative politics. Annual Review of Political, 2, 369-404. Disponible en: https://doi.org/10.1146/annurev.polisci.2.1.369.

Walker, E. T., McQuarrie, M. y Lee, C. W. (2015). Rising Participation and Declining Democracy. En C. W. Lee, M. McQuarrie y E. T. Walker (eds.). Democratizing Inequalities: Dilemmas of the New Public Participation (pp. 3-23). New York: NYU Press. Disponible en: https://doi.org/10.18574/nyu/9781479847273.003.0001.

Wampler, B. (2007). Can participatory institutions promote pluralism? Mobilizing. Social Science Quarterly, 41(4), 57-78.

- (2012). Participation, representation and social justice: Using participatory governance to transform representative democracy. Polity, 44 (4), 666-682. Disponible en: https:// doi.org/10.1057/pol.2012.21

Warren, M. E. (2009). Citizen participation and democratic deficits: considerations from the perspective of democratic theory. En J. De Bardeleben y J. Pammett (eds.). Activating the citizen: Dilemmas of participation in Europe and Canada (pp. 17-40). New York: Palgrave Macmillan. Disponible en: https://doi.org/10.1057/9780230240902_2. 


\section{ANEXO. Guía temática-Entrevistas con especialistas universitarios $y$ profesionales}

\section{PARTE I. Introducción}

\section{Presentación.}

— El tema: transformaciones, cambios, efectos de los PPs, cuáles permanecen tras los PPs / antes y después / más allá de los PPs.

- Presentación dinámica de la entrevista (grupo).

2. Experiencia personal de entrevistados en los procesos participativos.

- Trayectoria profesional / académica en el campo.

- Perspectivas, enfoques, preguntas que han centrado su interés o práctica.

- Experiencias concretas de PPs que conocen.

\section{PARTE II. Cambios observados después de los procesos participativos}

1. Cambios generales observados. [Antes y ahora] o [Causas/condiciones influyentes]

- Cambios positivos vs. negativos.

- Cambios esperados vs. no-esperados.

- Cambios duraderos vs. temporales / efímeros.

2. Cambios en la sociedad civil. [Antes y ahora] o [Causas/ condiciones influyentes]

— Cambios en objetivos.

- Sentimientos de identidad.

- Cambios hacia dentro: composición, organización y funcionamiento.

- Cambios hacia fuera: auto-percepciones respecto a las instituciones.

- Cambios observados en la forma en que la sociedad civil y la ciudadanía se relacionan:

- Cambios identificados.

- Cambios en los canales de comunicación.

- Cambios en la rendición de cuentas.

- Cambios en las relaciones entre organizaciones:

- Cambios.

- Fortalecimientos vs. debilitamiento de los contactos.

- Cooperación vs. competición.

- Cambios en la red de actores que interviene en los procesos de políticas públicas:

- Cambios.

- Nuevos objetivos.

- Nuevos conocimientos. 
.../...

3. Cambios en la relación entre la sociedad civil y la administración. [Antes y ahora] o [Causas/ condicionantes influyentes]

- Democratización.

- Empoderamiento de grupos.

- Variaciones en la conflictividad.

- Prácticas clientelares.

Conclusiones 